UTILIZAÇÃO DO MS-VISUAL STUDIO E DO ARDUINO PARA MEDIÇÕES DE TEMPERATURA

\title{
USING MS-VISUAL STUDIO AND ARDUINO TO DO TEMPERATURE MEASUREMENTS
}

\author{
LORSCHEITER, Thiago Aguirre; PAIM, João Paulo Silveira ${ }^{2}$; DE BONI, Luis Alcides Brandini ${ }^{*}$; \\ SILVA, Isaac Newton Lima ${ }^{4}$. \\ 1,2 Pontifícia Universidade Católica do Rio Grande do Sul, PUCRS. Faculdade de Engenharia, Controle e \\ Automação. Av. Ipiranga, 6681 - Prédio 30 - Bloco B - Sala 101. CEP: 90619-900 Porto Alegre - RS - Brasil.
}

${ }^{3,4}$ Pontifícia Universidade Católica do Rio Grande do Sul, PUCRS. Pós-Graduação em Engenharia e Tecnologia de Materiais, PGTEMA. Avenida Ipiranga, 6681 - Prédio 30 - Bloco D - Sala 107. CEP: 90619-900 - Porto Alegre - RS. Brasil.

${ }^{*}$ Autor correspondente

e-mail: labdeboni@gmail.com

Received 12 June 2011; received in revised form 27 June 2011; accepted 03 August 2011

\section{RESUMO}

Monitorar a variação da temperatura ao longo do tempo é uma tarefa importante, por exemplo, na análise dos resultados de muitas reações químicas. Neste trabalho foram desenvolvidos softwares para realizar a comunicação serial entre um computador $(P C)$ e o Arduino, visando o registro simultâneo da temperatura medida por dois termopares tipo $\mathrm{K}$ ao longo do tempo. A magnitude da temperatura adquirida pelos termopares, instrumentos analógicos, foi convertida para sinal no formato digital através de um chip MAX6675 e enviada para o computador através do Arduino via porta serial. No computador estes dados foram expressos graficamente em tempo-real através do aplicativo desenvolvido para esta finalidade e armazenados em arquivos compatíveis com planilhas eletrônicas para análises matemáticas posteriores.

Palavras-chave: Aquisição de dados, Arduino e MS-Visual Studio, Expressão gráfica de sinais analógicos, Medição de temperatura.

\section{ABSTRACT}

This work was carried out as an exercise in developing open source software to interface with free hardware. In this work a software was developed to perform serial communication between a computer (PC) and the Arduino, aiming at the simultaneous recording of two temperatures measured by type $\mathrm{K}$ thermocouples as a function of time. The temperatures measured by the analog instruments, thermocouples type K, were converted into digital signals through the MAX6675 and sent to the PC via the Arduino serial port. At the computer these data were expressed graphically in real time through the application developed for this purpose and then stored in files compatible with spreadsheets for subsequent mathematical analysis.

Keywords: Data acquisition, Arduino and MS-Visual Studio, Graphical expression of analog signals, Temperature measurement.

PERIÓDICO TCHÊ QUÍMICA • www.periodico.tchequimica.com • Vol. 8 N. 16. - ISSN 1806-0374 (impresso) • ISSN 1806-9827 (CD-ROM) • ISSN 2179-0302 (meio eletrônico) 


\section{INTRODUÇÃO}

Monitorar a variação da temperatura ao longo do tempo é uma tarefa importante, por exemplo, na análise dos resultados de muitas reações químicas é possível entender por que um determinado produto não foi sintetizado ao observar-se que a reação foi conduzida na temperatura errada.

Este artigo descreve a utilização do MSVisual Studio em conjunto com a plataforma de hardware livre Arduino, visando o monitoramento de dois sensores de temperatura termopar tipo $\mathrm{K}$ (cromel-alumel). E a construção do aplicativo de código aberto Lorscheiter Viewer 1.0. Para tanto é necessário certo conhecimento prévio de linguagem de programação .NET (Pandey e Nathan, 2002) e C/C++ (Davis, 2004).

Em virtude do termopar tipo $\mathrm{K}$ ser formado pela união de duas ligas metálicas cromel-alumel, compostas respectivamente de $90 \% \mathrm{Ni} \mid 10 \% \mathrm{Cr}$ e $95 \% \mathrm{Ni} \mid 5 \% \mathrm{Al}$ (Omega, 1999; Thermometrics Corporation, 2011), para aumentar a vida útil do instrumento, é relevante evitar a introdução deste tipo de termopar em ambientes incompatíveis com Al, $\mathrm{Cr}$ e especialmente Ni (Fisher Scientific, 2011; Oxford University 2004).

\section{MATERIAL E MÉTODOS}

\subsection{Lista de materiais e softwares:}

- 1 computador com o sistema operacional MS-Windows 7 (ou equivalente) ${ }^{1}$;

- Software MS-Visual C\# 2010 Express $^{2}$ ou MS-Visual Studio ${ }^{3}$;

- Software Arduino 00224,

- Software Easily Applicable Graphical Layout Editor ${ }^{5}$ (EAGLE,5.10.0);

- Software. NET Framework 4.06

- MAX6675 Arduino library code ${ }^{7}$;

- 1 Arduino Mega 1280;

- 2 termopares tipo K;

- 2 shields com 0 conversor A/D

1 Vide referência do MS-Windows 7 .

2 Vide referência do MS-Visual C\# Express

3 Vide referência do MS-Visual Studio 2010.

4 Vide referência do Arduino 0022.

5 Vide referência do EAGLE 5.10.0

6 Vide referência do .NET Framework 4.0

7 Vide referência do MAX6675 Arduino library code
MAX6675;

- Cabos e conectores em quantidade suficiente para;

- Equipamento para confecção de circuitos elétricos;

\subsection{Preparando o MAX6675}

O datasheet do componente MAX6675 é um manual com características e parâmetros para a utilização do MAX6675 (Campillo, 2011) e sua leitura é recomendável para evitar danificar o componente.

O circuito com o MAX6675 pode ser manufaturado localmente após a compra dos componentes requeridos ou adquirido de um fornecedor comercial (Adafruit Industries, 2011). A função do MAX6675 é amplificar e converter o sinal analógico, gerado pelo termopar tipo-k, para um sinal digital de 12 bits que o microprocessador do Arduino é capaz de fazer a leitura via protocolo SPI (Serial Peripherical Interface (Pérez, 2004)) da temperatura captada. O fabricante do componente fornece um pequeno circuito elétrico para alimentação e redução do ruído, conforme Figura 1.

A placa de circuito impresso pode ser manufaturada através de técnica substrativa, como a serigrafia (silk screen printing), que estava disponível no laboratório (Jones 2004; Barbosa, 2006; Simões, 2008). Os componentes utilizados na construção da placa de circuito impresso estão disponíveis comercialmente. O roteamento da placa foi feito com o software Eagle 5.10.0 (Krug, 2011).

A medida de temperatura efetuada através dos termopares em um meio qualquer é enviada ao Arduino através de uma placa com um conversor analógico-digital (A/D) MAX6675 (Maxim, 2011; Campillo, 2002). Os dados relativos a temperatura, recebidos pelo Arduino, são enviados no formato decimal para um computador através da porta serial.

\subsection{Configurando o Arduino MEGA}

O Arduino MEGA deve ser conectado ao computador e o Código 1, adaptado de Limor (2011), Fuller (2011) e Moussette (2011), deve ser inserido no Ambiente de Desenvolvimento Integrado do Arduino (IDE - Integrated Development Environment), conforme a Figura 2. 
Após inserir o código 1 no IDE do Arduino, o código deve ser enviado ao Arduino através do botão Upload (da IDE). Esta parte esta completa.

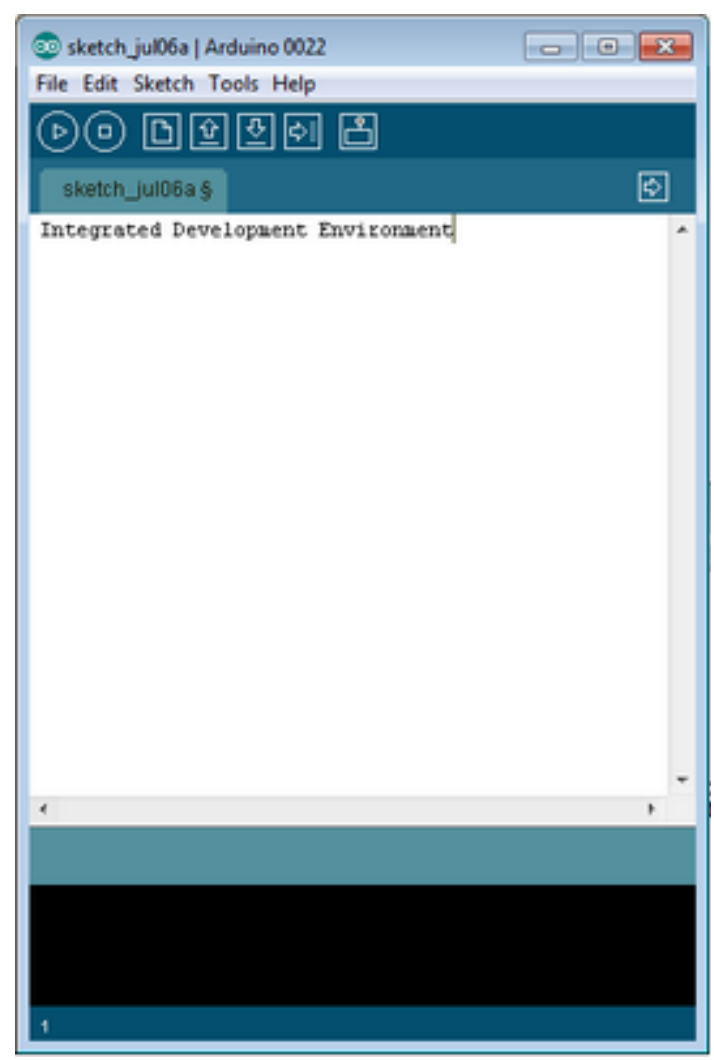

Figura 2. Vista do IDE do Arduino.

\subsection{Preparando o software Lorscheiter Viewer}

O aplicativo Lorscheiter Viewer 1.0. foi desenvolvido para apresentar graficamente os valores que o Arduino envia ao computador através da porta serial USB, e armazenar os mesmos de forma organizada para posteriores análises matemáticas. Este software é distribuído sob a licença GNU (2007).

O software Lorscheiter Viewer é compilado no MS-Visual Studio. Para isto basta abrir o código fonte disponível no site do aplicativo na IDE do MS-Visual C\# 2010 Express ou do Visual Studio. O software deve ser compilado para uso e distribuição. A aparência do software é similar à Figura 3.

\subsection{Unindo todas as partes}

Após montar, ou adquirir, a placa com o conversor A/D MAX6675 e conectar o mesmo ao Arduino (Figura 4) e esta pronto para executar medições de temperatura em diferentes ambientes.

Na Figura 4, o conversor está conectado ao Arduino através dos pinos PWM (Pulse Width Modulation) 2 a 7 e a segunda placa com 0 conversor MAX6675 é conectada ao Arduino, conforme a programação do Código 1, através dos pinos 8 a 12 .

\section{RESULTADOS E DISCUSSÃO}

Este experimento permitiu a montagem de um sistema de aquisição de dados que monitora e armazena a variação da temperatura através de dois sensores termopar tipo k. Sem proteção adicional aos termopares, as medições podem ser executadas em qualquer meio que não ataque quimicamente a liga metálica cromelalumel.

Os sensores de temperatura podem ser substituídos por outros instrumentos de medição, desde que o código do Arduino seja devidamente atualizado e envie outros dados para o Lorscheiter Viewer no formato decimal através da porta de comunicação. Os dados devem manter a forma de um vetor, conforme o modelo apresentado por Fuller (2011).

Após o término do processo substrativo de preparo da placa do MAX6675, seus componentes foram soldados na mesma conforme a Figura 5.

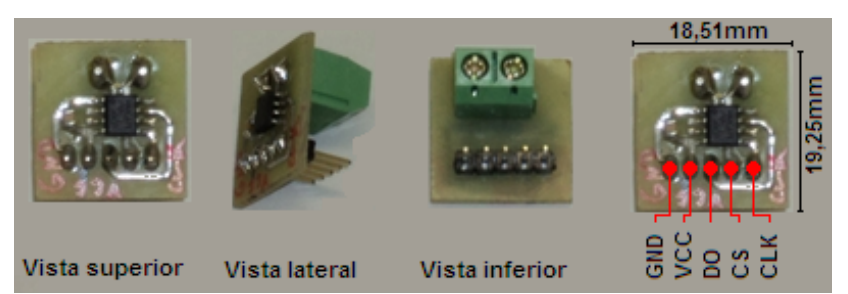

Figura 5. Placa do MAX6675, onde GND = Ground, VCC $=$ Common-collector voltage, $\mathrm{DO}=$ Data out, $\mathrm{CS}=$ Chip select e CLK = Clock 


\section{CONCLUSÕES}

A utilização de softwares e hardwares livres provém meios rápidos e práticos para a execução do monitoramento de parâmetros físicos. Neste trabalho isto possibilitou a construção de um instrumento para monitorar a variação da temperatura através de dois termopares tipo k. O mesmo instrumento também pode ser utilizado para monitorar a temperatura de reações químicas compatíveis.

Os softwares desenvolvidos para este trabalho (Lorscheiter Viewer e ARDUINO L.D.A.) podem ser utilizados independentemente um do outro, todavia é interessante sua utilização em conjunto, em virtude da facilidade do gerenciamento dos dados obtidos através do sistema de monitoração. Ambos softwares são distribuídos sob a licença GNU.

\section{AGRADECIMENTOS}

Os autores agradecem a PETROBRAS e ao CNPq. Ao programa Microsoft Developer Network (MSDN) Academic Alliance. Ao Laboratório de Processos Ambientais (LAPAPUCRS).

\section{REFERÊNCIAS}

1. Campillo, Fe. MAX6675 DS. Disponível, em: $\quad<$ http://datasheets maximic.com/en/ds/MAX6675.pdf>. Acesso em jul 2011.

2. MAXIM. MAX6675 Cold-JunctionCompensated K-Thermocouple-to-Digital Converter $\left(0^{\circ} \mathrm{C}\right.$ to $\left.+1024^{\circ} \mathrm{C}\right)$. Disponível, em <http://www.maximic.com/datasheet/index.mvp/id/3149>.

Acesso em jul 2011.

3. http://windows.microsoft.com/ptBR/windows/home, acessada em Julho 2011.

4. http://www.microsoft.com/visualstudio/ptbr, acessada em Julho 2011.

5. http://arduino.cc/en/Main/Software, acessada em Julho 2011.

6. Free Software Foundation. The GNU General Public License v3.0. $<$ http://www.gnu.org/licenses/gpl.html>, acessada em Julho 2011.
7. FULLER, Lynn. Graphing sketch for multiple analog signals. Disponível em $<$ http://people.rit.edu/lffeee/Processing_Di splay_Analog_Signal_Fuller.pde>. Acesso em Jul. 2011.

8. MAX6675 Arduino library code. $<$ https://github.com/adafruit/MAX6675library>, acessada em Julho 2011.

9. Limor. Thermocouple sensor tutorial. Disponível em: $<$ http://www.ladyada.net/learn/sensors/the rmocouple.html >. Acesso em jul 2011.

10. MOUSSETTE, Camille. Sensors, serial communication and data logger. Disponível em $<$ http://www.interactiondesign.se/wiki/cour ses:2010.12.01.intro_prototyping >. Acesso em jul 2011.

11. http://www.cadsoft.de/downloads/, acessada em Julho 2011.

12. BARBOSA, Luís Antônio de Araújo. A tecnologia de fabricação de placas de circuito impresso. Disponível em <http://bt.fatecsp.br/system/articles/528/or iginal/48.pdf>. Acesso em Jul 2011.

13. JONES, David L. PCB Design Tutorial. Disponível em $<$ www.alternatezone.com/electronics/files/ PCBDesignTutorialRevA.pdf>. Acesso em Jul 2011.

14. SIMÕES, Luis. Desenho Electrotécnico. Disponível em <http://www.estv.ipv.pt/PaginasPessoais/ eduardop/Desenho/Acetatos/Desenho $\% 20$ Electrotécnico\%20\%20Apresentação_.pdf>. Acesso em Jul 2011.

15. DAVIS, Stephen Randy. C++ for Dummies. 5Ed. John Wiley \& Sons, Inc.432p.

16. PANDEY, Nitin; NATHAN, Senthil. Visual Studio.Net All in One Desk Reference for Dummies. 1Ed. John Wiley \& Sons, Inc. 960p.

17. KRUG, Rodrigo. CadSoft Eagle 5.10, Uma Aplicação Prática. Disponível em $<$ http://www.ee.pucrs.br/ rodrigok/reposit orio/mc_usp/Tutorial_Eagle_Final_pt.pdf> . Acesso em Jul 2011.

18. Adafruit Industries. Thermocouple Amplifier (MAX6675) breakout board v1.0. Disponível em <http://www.adafruit.com/products/269>. Acesso em Jul 2011. 
19. http://www.microsoft.com/visualstudio/enus/products/2010-editions/visual-csharpexpress, acessada em Julho 2011.

20. http://lorscheiterviewer.blogspot.com/, acessada em Julho 2011.

21. Fisher Scientific. Material Safety Data Sheet Nickel Metal. Disponível em <http://fscimage.fishersci.com/msds/1624 0.htm>. Acesso em Jul 2011.

22. Oxford University. Safety data for Nickel. Disponível em $<$ http://msds.chem.ox.ac.uk/NI/nickel.html >. Acesso em Jul 2011.

23. Omega. Revised Thermocouple Reference Tables Type K. Disponível em <www.omega.com/temperature/zl pdf/z204-206.pdf>. 1999. Acesso em Jul 2011

24. Thermometrics Corporation. TYPE K THERMOCOUPLE (Chromel I Alumel). Disponível em <http://www.thermometric scorp.com/thertypk.html>. Acesso em Jul 2011

25. http://msdn.microsoft.com/pt-br/netframe work/default.aspx, acessada em Julho 2011.

\section{Código 1 (Parte do Arduino)}

\section{/*}

ARDUINO L.D.A. (Leitor de Dados do Arduino) 1.0. (Julho, 2011)

Este programa envia dados de vários sensores através da porta serial em 9600 bauds na forma de vetores para serem graficamente representados em um PC pelo programa Lorscheiter Viewer 1.0 (ou superior ou equivalente)

Referências do programa estão disponíveis em: *http://www.ladyada.net/learn/sensors/thermocouple. html

*http://people.rit.edu//ffeee/Processing_Display_Anal og_Signal_Fuller.pde

*http://www.interactiondesign.se/wiki/courses:2010.1 2.01.intro_prototyping

Bonis

Copyright (C) <2011> < Luis Alcides Brandini De

labdeboni@gmail.com

This program is free software: you can redistribute it and/or modify it under the terms of the GNU General Public License as published by the Free Software Foundation, either version 3 of the License, or any later version.

This program is distributed in the hope that it will be useful, but WITHOUT ANY WARRANTY; without even the implied warranty of MERCHANTABILITY or FITNESS FOR A PARTICULAR PURPOSE. See the GNU General Public License for more details.

You should have received a copy of the GNU General Public License along with this program. If not, see <http://www.gnu.org/licenses/>.

$$
\text { *I }
$$

//The comments inside the code are not translated yet. \#include "max6675.h" //chama a biblioteca do MAX6675, que foi previamente //instalada na pasta "libraries" do IDE do

Arduino.

//Primeiro termopar

int thermoDO = 4; //DO (Saída de dados)

int thermoCS $=5 ; / / C S$ (chip select)

int thermoCLK $=6$; //CLK (Relógio)

// Segundo termopar

int athermoDO = 10;

int athermoCS $=11$;

int athermoCLK $=12$;

MAX6675 thermocouple(thermoCLK, thermoCS, thermoDO);// termopar 1

//Primeiro termopar

int vccPin $=3$;

int gndPin $=2$;

MAX6675 athermocouple(athermoCLK, athermoCS, athermoDO);// termopar 2

// Segundo termopar

int avccPin $=9$;

int agndPin $=8$;

void setup() \{

Serial.begin(9600);

// Configura os pinos do Arduino como GND e VCC

pinMode(vccPin, OUTPUT); digitalWrite(vccPin,

HIGH);

pinMode(gndPin, OUTPUT); digitalWrite(gndPin, LOW);

pinMode(avccPin, OUTPUT); digitalWrite(avccPin, HIGH);

pinMode(agndPin, OUTPUT); digitalWrite(agndPin, LOW);

Serial.printIn("MAX6675 Carregando....");

// Aguarda a estabilização do chip MAX6675

delay(1000); //espera 1 segundo para atualizar os 
dados

\}

void $\operatorname{loop}()\{$

Serial.print(thermocouple.readCelsius());// termopar \} 1

Serial.println( );
2

Serial.print(athermocouple.readCelsius());// termopar

Serial.println( );

delay(1000);

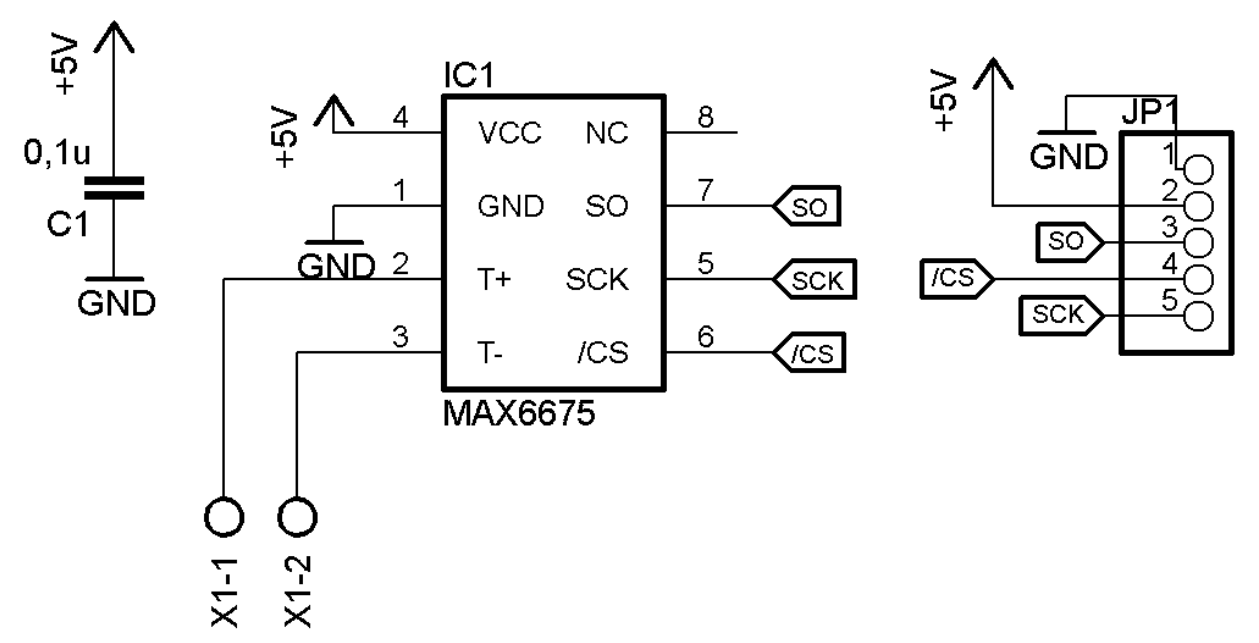

Figura 1. Circuito elétrico para alimentação e redução do ruído do MAX6675

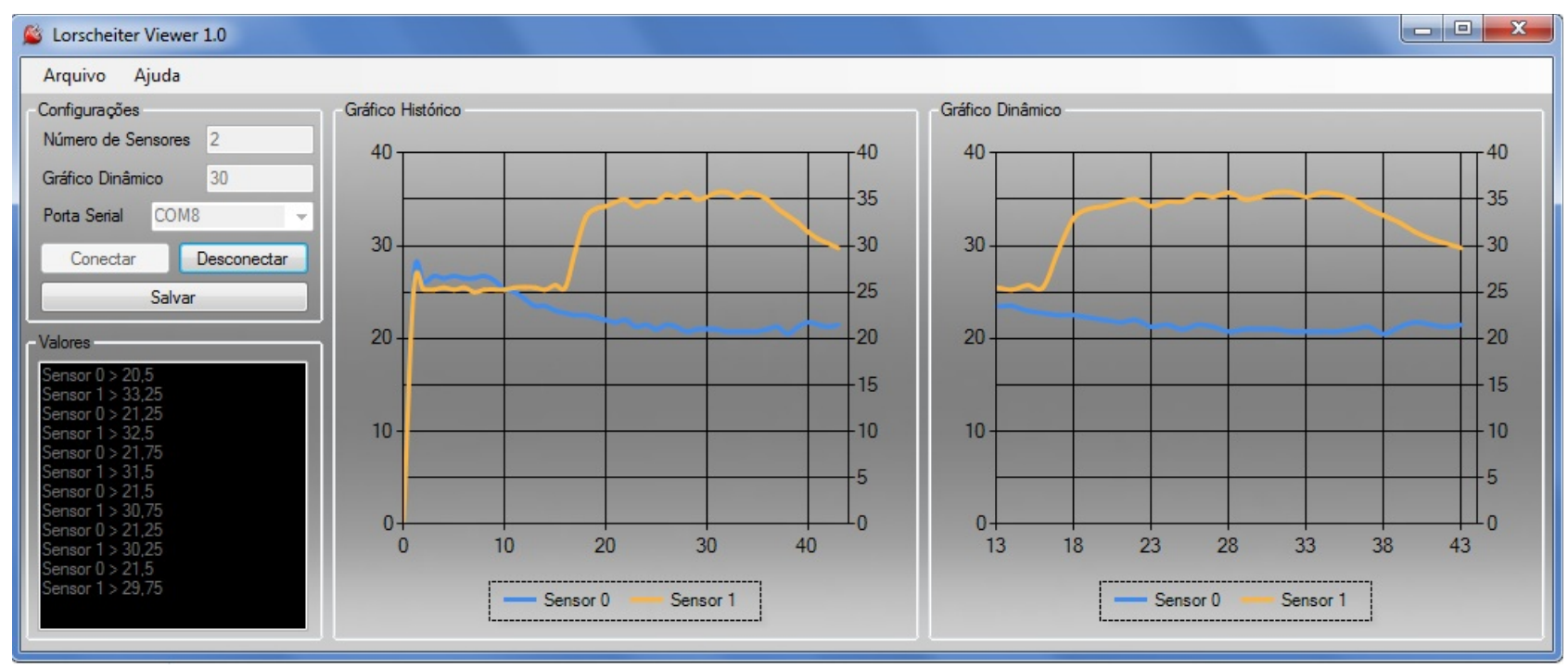

Figura 3. Print Screen do software Lorscheiter Viewer 1.0

PERIÓDICO TCHÊ QUÍMICA • www.periodico.tchequimica.com • Vol. 8 N. 16. •ISSN 1806-0374 (impresso) • ISSN 1806-9827 (CD-ROM) • ISSN 2179-0302 (meio eletrônico) 


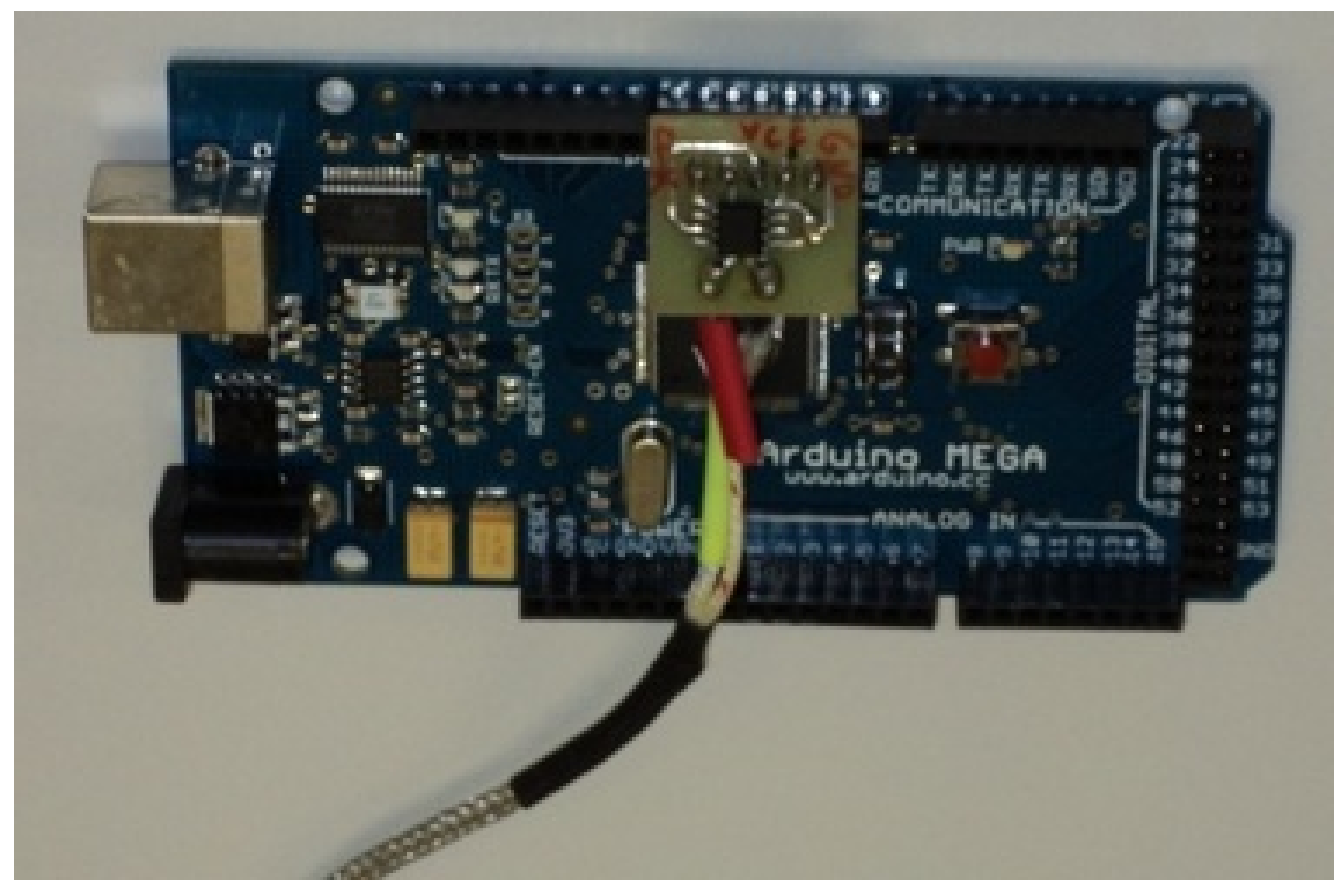

Figura 4. MAX6675 conectado ao Arduino Mega através dos pinos PWM 2 a 7. O segundo termopar é conectado através dos pinos 8 a 12 .

PERIÓDICO TCHÊ QUÍMICA • www.periodico.tchequimica.com • Vol. 8 N. 16. • ISSN 1806-0374 (impresso) • ISSN 1806-9827 (CD-ROM) • ISSN 2179-0302 (meio eletrônico) (C) 2011. Porto Alegre, RS. Brasil 